\title{
New Organizational Forms for Information Security Management
}

\author{
Richard Baskerville \\ Copenhagen Business School and Binghamton University \\ Binghamton, New York 13902-6015, Tlf. +1 607777 2337, \\ Fax +1 6077774422 , Email rbask@binghamton.edu
}

\begin{abstract}
This paper contributes a functional analysis of current forms of information security management. This analysis is then applied in a discussion of organizational options for information security management including organizational location, organizational schemes (such as hierarchies, matrices and networks) and degree of outsourcing. Newer hybrid organizational forms are increasingly important for the development of distributed security management.

Keywords

Computer Security Management, Information Systems Security Management, New Organizational Forms, Disaster Recovery Planning, Contingency Planning
\end{abstract}

\section{INTRODUCTION}

The paradigm for organization and operation of the information security functions is typically centralized and rigid ( $c f$. Forcht 1994). This has been reasonable in the past given the mission of information security management. However, newer forms of organizations are evolving in which the information systems (IS) function is distributed in various ways (Smithson et al 1994). Motives for such distribution include organizational transformation (Applegate 1994) and the rationalization of business processes (Hammer and Champy 1993). These newer forms of organization are placing demands for flexible and adaptive security management (Baskerville 1993). The need for distributed and flexible information security management is a considerable paradigm shift for most information security managers.

However, before we can discuss the different ways in which the organizational security function can be structured, we should first survey and analyze the major 
functional elements of this organizational component. After this functional survey, we will discuss organizational structures that might be used to plan, coordinate and control organizational information systems security in newer organizational forms. The exact activities related to each of these management functions are beyond the scope of this paper. Detailed discussions would require a book-length treatise, since these detail the complete IS security function. However, brief summary indicators are mentioned for each of these functions.

\section{FUNCTIONS OF INFORMATION SYSTEMS SECURITY}

The following analysis raises a hierarchical inventory of the essential functions of an organization's information systems security element. While an information systems security department could be structured in this manner, these functions are more often distributed across different organizational lines rather than concentrated in a single organizational department.

\section{Operation Functions}

The operation functions deal with the day-to-day, routine operation of security safeguards.

Media Backup Operation. This operation regards the routine writing of backup file tapes, the indexing of these files and tapes, and the shipment of these tapes to off-site storage locations.

Incident Monitoring. Monitoring is a routine security control function that captures information about both major and minor security incidents. Although most incidents have no connection with potential disasters, incident monitoring is a key coordination element in disaster recovery.

Anomaly Investigation. Systems anomalies may arise in the form of errors or unexplained erratic behavior in the information system. Usually these will lead to the discovery of errors or faults in the system hardware or software.

Information Classification. If the organization maintains a classification structure for sensitive information (e.g., "secret" or "confidential"), a security function should exist to record and monitor the use of the structure.

Access Control. The access control function involves managing access accounts, data base access control, authentication token (e.g., smart card) management, firewall and sucker trap operation and intrusion monitoring.

Safeguard Operation. The security operation function is also responsible for the operation of any other security safeguards such as management of cryptographic keys, logging computer room visitors or recording serial numbers of equipment on loan. 


\section{Security Development Functions}

Another major functional area that should be coordinated by information security management is the development of security.

Policy Development. General security policies and procedures provide the framework for much of the non-computer-based information security, e.g., rules for classifying information, storing types of documents, and the rewards and sanctions structure related to personnel involved in security incidents.

Safeguards Design regards the activities involved in analyzing and specifying the safeguards necessary in various application systems in the organization's applications portfolio.

Safeguards Implementation regards the activities involved in constructing, acquiring, installing and testing the safeguards necessary in various application systems in the organization's applications portfolio.

Security Operations Auditing. Audits of information systems usually involve an unannounced inspection of security safeguards in order to verify that these are being used correctly. Examples of such audit activities include EDP auditing and tiger team operation (a tiger team consists of security specialists who undertake the task of "attacking" an information system as if they were computer criminals).

Security Design Auditing. Auditing of general systems analysis and design processes and products determines the compliance of systems analysts and designers and their specifications with the organization's standard methods for conducting such projects.

\section{Human Resources Functions}

Many of the information systems security activities regard the people who are involved in processing organizational information, and using that information in their decision making.

Security Awareness and Training. A security training program has the goal of enabling people to use the systems safely by teaching them how to use the security features correctly.

Disaster Recovery Drills have two major goals. One is to test the technical feasibility of plan resources such as backup sites and media. This second goal is the development of disaster recovery competence in the potential members of a crisis management team and its support staff.

Security Clearance. If the organization has an information classification system, there should be a policy of clearance assignments for the people in the organization authorized to used classified information. IS security must manage these clearances or coordinate with other managers in charge of such clearances.

Electronic Employee Monitoring. Computer-based information systems have enabled a widespread number of electronic monitoring activities within systems applications. Some of these activities (e.g., intrusion detection) may fall under IS security management. 


\section{Strategy and Planning Functions}

Most of the other information systems security functions discussed above may be under the operational control of other departments, and may only be subject to coordination with the department tasked with managing information systems security. In contrast, the strategic and planning functions will fall squarely under the direct responsibility of security management.

Methodology Research. IS security involves specification of systems development and deployment methods that have sound security elements.

Risk Analysis. The security planning function will usually be responsible for evaluating the overall organizational IS risk profile.

Disaster Recovery Planning. The information system security management function usually takes direct responsibility for disaster recovery (business continuity or resumption) planning, since information technology (IT) is one of the most problematic elements of such recovery.

Assurance. Planning the general information risk posture includes adjusting the strategies regarding the organization's insurance portfolio. Coordination of the insurance portfolio with the security program is critical because changes in the insurance details will sometimes affect the degree of risk to information assets.

Security Program Planning. An organization's entire information systems security program must be coordinated and planned, and might consist of all of the coordinated activities outlined above. From this perspective, this analysis not only describes the functions that must be coordinated by the information security element, it also describes the possible components that might be developed by an information systems security program.

\section{COORDINATION AND STRUCTURAL OPTIONS}

In the previous section, we surveyed the various functions that must be coordinated by information systems security management. This section provides a survey and an analysis of ways in which the coordination of these functions might be structured. There are three dimensions used in this analysis: functional location, organizational scheme, and degree of outsourcing.

\subsection{Locating The Information Systems Security Function}

The information systems security function is usually considered a staff function unless the information system is producing the main (signal) output product of the organization. The location of the information security elements within the organization will probably be a function of how critical the information systems security needs are to the objectives and goals of the organization. Figure 1 illustrates a few possible locations for the IS security function. These are briefly discussed below, classified according to the related strength (risk) of information system hazards facing the organization. 


\section{(Under CEO)}

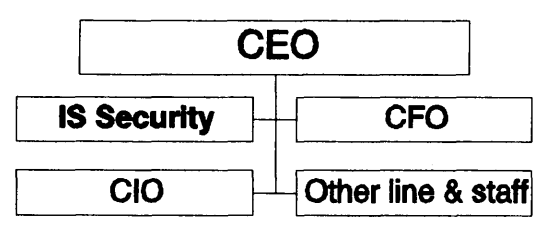

(Under CFO)

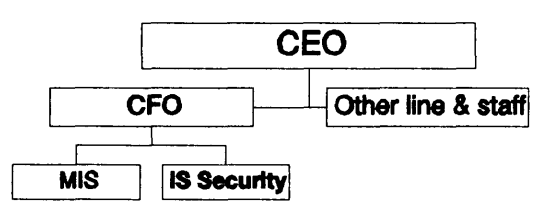

(Under EDP Auditing)

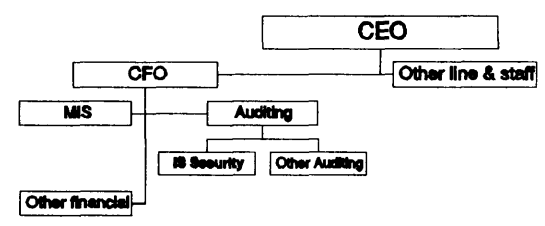

(Under $\mathrm{ClO}$ )

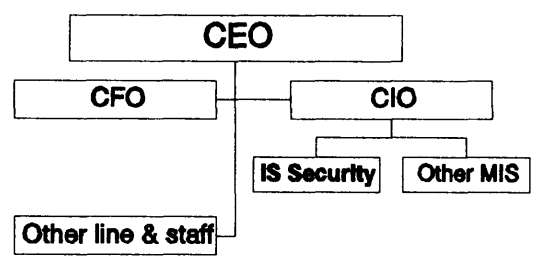

(Under MIS)

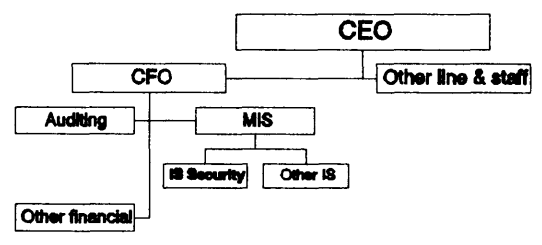

(Under CSO)

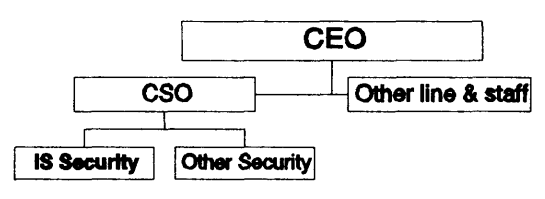

Figure 1. Some possible organizational locations for the IS security function.

\section{Strong Hazard Level}

If the security risks are high and the information systems are extremely important to the organization, as in military, diplomatic or financial institutions, then the security element may be located near to the chief executive officer (CEO). If the systems are somewhat less critical and the risks lower, then the function might be located on a parallel with the management information systems function (MIS), for example under the chief financial officer (CFO). These locations have the advantage of high visibility to senior management and represent an extremely strong organizational commitment to information security. The disadvantage lies in the separation of the functional IS management and security management, raising possibilities for fairly high-level strategic conflicts between IS and its security. 


\section{Moderate Hazard Level}

If the information systems are not extremely critical and the risks are moderate, then the information security function will probably be placed under the chief information officer (CIO) or attached to the information systems department. The advantage of locating IS security within the IS group is the moderation of conflict between IS functionality and security, since the same management coordinates both elements. The disadvantage lies in the difficulty of preserving the rather transparent benefits of security safeguards under cost-overrun and behind-deliveryschedule pressures. Often the IS management rewards functional delivery above all other criteria, including (unfortunately) safety.

\section{Weak Hazard Level}

In low-risk settings, a possible alternative location groups the information security coordinating function with related security activities. For example, the IS security function might be grouped with the internal auditing function or under the chief security officer (i.e., corporate security). The advantage in this location arises in the improved technical specialization of the security professionals and better efficiency in their tasks. The disadvantage lies in a strong emphasis on detection rather than prevention of the consequences of various information system hazards.

\subsection{Organizational Scheme}

Regardless of where the IS security organization is located, it should retain coordination responsibilities over the IS security functions analyzed earlier. However, with the possible exception of a few IT-intensive organizations with very severe security needs, it is unlikely that the IS security department would retain direct authority over all of the security functions. It is more likely that this department would retain direct authority over some part of these functions, with an appropriate staff, facility and budget to support these activities. The remaining functions would be distributed among other cognizant organizational groups, and the IS security department would retain only the authority to plan, monitor, evaluate and otherwise coordinate the necessary IS security activities of the other departments. As is the case for other modern organizations, there are three basic schemes (the hierarchy, the matrix, and the network) for organizing IS security.

\section{Hierarchy}

Figure 2 illustrates a possible IS security organizational structure as an hierarchical organization. This organization consists of five major functions much as described earlier in this paper. The human resources activities are focussed on disaster recovery drills and security awareness and training. (Other human resource activities are either not present in this organization, or directly managed by other organizational functions, e.g., personnel or production management.) Similarly the security development activity is focussed on auditing designs and operations while safeguards design and implementation is not directly managed by the IS security function, and is probably under the direct control of other elements in the 
organization (e.g., the MIS department). In this particular organization, the IS security department has grouped a select number of specialist activities under the rubric "special operations". These include investigations of system anomalies, incident monitoring, firewall programming and a tiger team activity. A staff operation, "security operations coordination", liaises with other departments to monitor and coordinate other elements in the security program and is likely to include most of the other missing activities (inventoried in the earlier functional analysis) that are not found in Figure 2.

The advantages of hierarchical organizations lie in their functional specialization and strong centralized control. The chief disadvantage is the rigidity that enables this specialization and control. These organizations are very efficient and reliable at performing routine functions over long periods, e.g., a lowtechnology manufacturing industry with high volume manufacturing runs. These organizations are not very flexible when it comes to adapting to change. In the context of the information security function, hierarchical organizations are appropriate in stable settings because of their efficiency and strong emphasis on high standards of technical performance.

\section{Matrix}

Figure 3 shows an alternative scheme for organizing the IS security function based on a matrix, project or grid organizational structure. A matrix organization offers some opportunity for distributing the IS security function. In this type of organization, the IS security function retains only operational control of security program planning and strategy. The operational security work is housed in the various MIS departments, such as computer operations, data base administration, network management, etc. However, a member of the coordinating IS security staff activity is involved in project teams tasked to carry out work within the MIS departments. These project teams are represented by the dark lozenges in Figure 3.

The advantage of matrix organizations lie in their improved adaptability while maintaining some degree of centralized control and technical specialization. Their disadvantage lies in the goal conflicts that arise from split management directions, and the inefficiency of maintaining multiple management structures.

Matrix organizations may be good compromises for flexibility in moderate and high risk settings (even when the underlying organization follows a hierarchical model). Basically this matrix would be constructed by mapping the major functions from the IS security hierarchy onto the normal IS hierarchy. The matrix compromise enables the information security organization to recognize and adapt to changes in its encompassing host organization.

\section{Network}

A third example of a scheme for the IS security function organization is newer: a network, virtual or "spaghetti" organization. This is a relatively new scheme that is typically dependent on special computer groupware to provide on-line meetings, shared databases, ad-hoc data mining and electronic communication. There are two new aspects to the security function within network organizations: (1) the increased reliance on information technology as an essential organizational enabling factor 


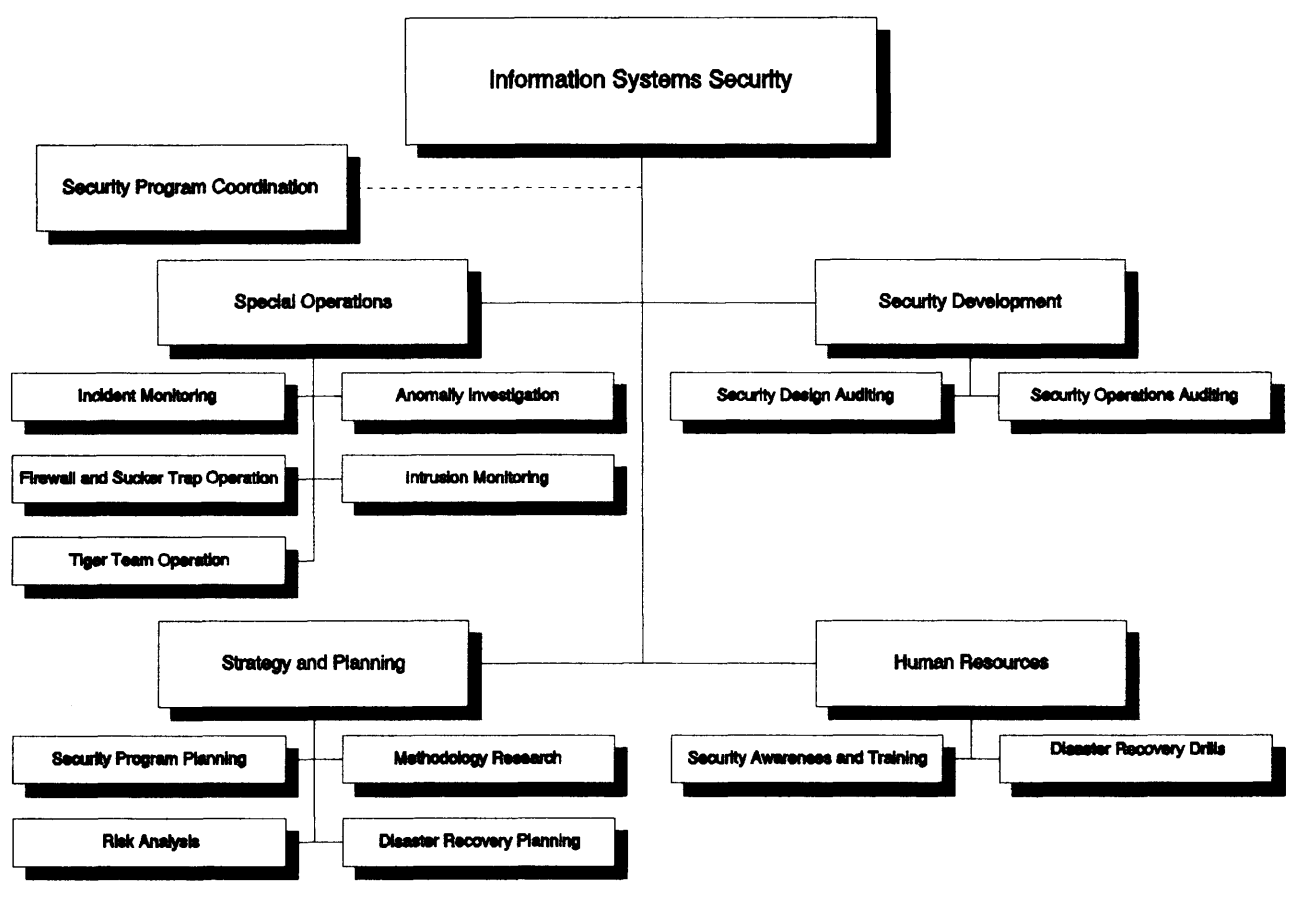

Figure 2. Example of IS Security Organization.

for such organizational structures; and (2) the devolution of organizational control from centralized management to entrepreneurial, cross-functional teams. The safety of the IT is more critical since these organizations are otherwise impossible. without newer IT. Totally centralized information security management is made problematic by devolved organizational control.

Network organizations focus on problem-solving accomplishments rather than routine tasks. While a hierarchical or network organizational outline may exist, the real form is kept fluid. When problems arise, ad-hoc groups form, solutions are implemented, and then the groups dissolve. Routine tasks are passed around cooperatively according to shifting group workloads. A network organizational form in security means that the roles of security professionals become blurred. Their tasks include attacking whatever problems arrest them, either by being brought deliberately to their attention by a co-worker, or by their own notice and interest. Importantly, the organization's departmental lines are also blurred. This means that security "outsiders" will be freely co-opted into the security organization according to interests and needs. Similarly, security specialists may get co-opted into "outside" tasks and problems.

The advantages of network organizations are their extremely high adaptability to change and their quick response to fast changing environments. The disadvantages include the heavy loss of efficiency and control, and the necessity for an expensive staff. Network organizations depend heavily on a well-motivated, creative and bright staff that seizes the initiative wherever necessary. Wherever the staff falter, the matter is difficult for management to detect and correct. 


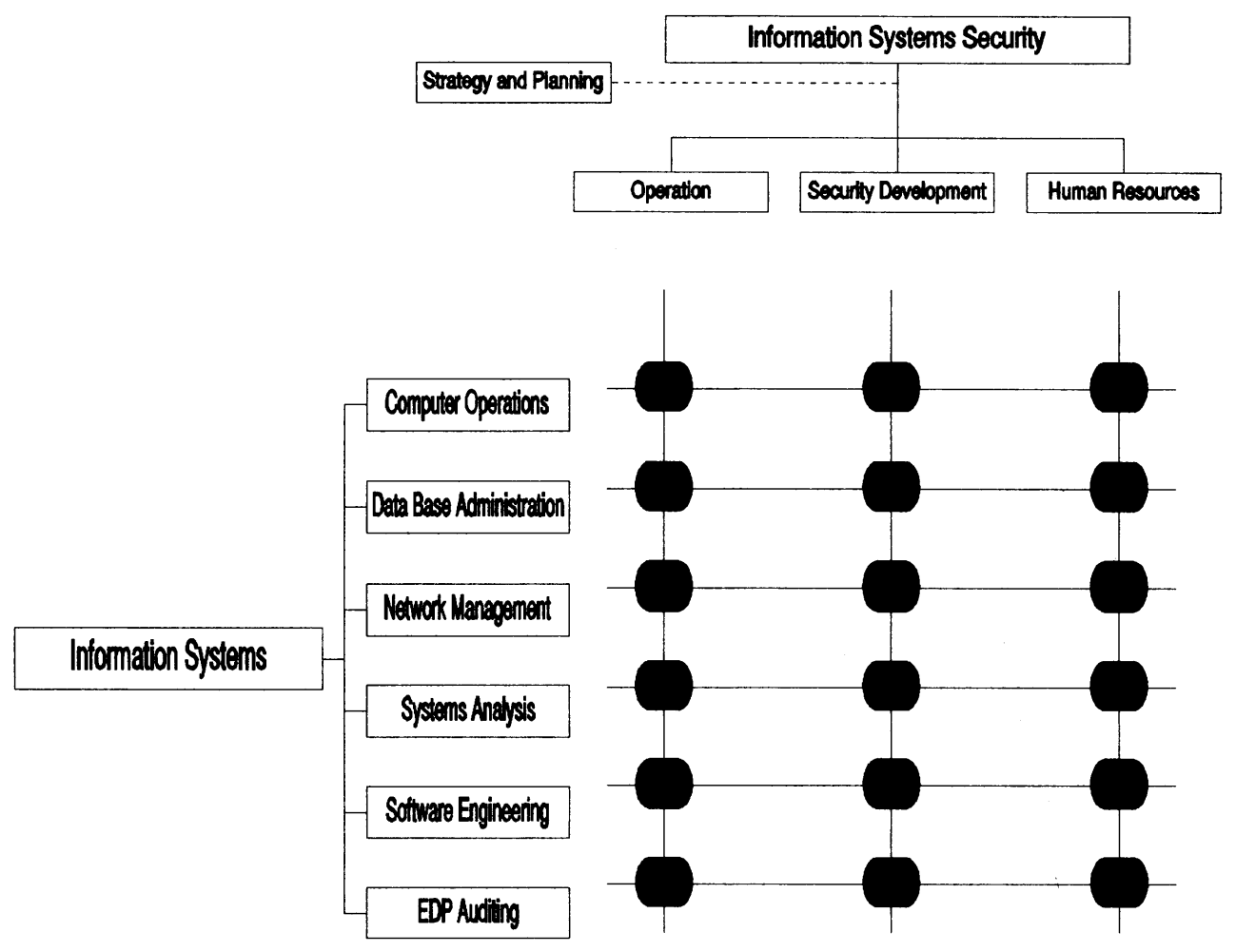

Figure 3. IS Security Management using a matrix organization.

Network organizations in information systems security are probably the only good response in an organizational setting that is network-based. This response is required in order to provide security support in a manner that is flexible enough to keep up with the shifting organizational requirements. However, if the organizational setting is highly volatile, a network organizational form may be suitable for the security organization, even if the host organization takes on a hierarchical or matrix form.

\subsection{Degree of Outsourcing}

Information security functions, and their underlying IS functions may be outsourced (contracted to an external organization) to a larger or smaller degree. The extent of this outsourcing has major effects on the organizational form of the information security element.

Outsourcing is not a new concept in information security management. Many organizations in moderate- or low-level hazard risk settings depend heavily on contractors and consultants for security expertise. While there may be a manager with security responsibilities in such organizations, virtually all of the security functions will be distributed among other departments. These organizations will 
bring in consultants for most specialist activities like security program planning, risk analysis and security design. In such settings, the chief operational duty of the IS security group will often be limited to security training and awareness programs.

The primary advantage of outsourcing is the high degree of technical expertise achieved at a low cost (compared to under-utilization of such expertise when maintained internally). The disadvantages revolve around the loss of control over outsourced functions. This loss raises such problems as the conflicting goals of contracted staff, varying availability of external resources, and the lack of internal expertise for strategy development.

The organizational security form must change if any part of the basic organizational information system has been outsourced. Essentially, all of the above-mentioned security functions must be coordinated with the external organization operating under the outsourcing contract. This will typically imply an underlying network or matrix organizational scheme that operates across the two organizations. The degree of overlap and coordination will vary according to the degree of outsourcing. For example, if there is total IS outsourcing, the amount of security coordination is minimal and the need for a network security organizational scheme would be unaffected by the outsourcing. Likewise, total insourcing of IS would minimize this motive for a network scheme. However, as the degree of outsourcing varies from zero to total the related demand for a network scheme would also vary (maximized, perhaps, around an even split between insourced and outsourced IS activity). A matrix organization might be more suitable for moderate outsourcing settings where technical expertise and review is critical, e.g., settings where IS development is outsourced, but IS operation is insourced.

\subsection{Hybrid Organizational Forms}

The various elements of organizational forms discussed above are idealized to a large degree. It is unlikely that an information security organization will implement all of these functions using a pure form of the coordination and structural options as described here. Most information security organizations will be a hybrid that incorporates a subset of these functions (probably with some different delineations) with a combination of several locations, several schemes and several degrees of outsourcing. For example, the security organization may follow a hierarchical scheme regarding its insourced IS operations, a matrix scheme regarding its IS development activities and a network scheme regarding its outsourced IS activities.

Figure 4 diagrams the dimensional analysis presented above and can be used to illustrate the hybrid nature of today's IS security organizational forms. The horizontal axis of this diagram maps organizational schemes moving from less flexible schemes on the left to more flexible schemes on the right. The vertical axis maps organizational location moving from those suitable to less critically hazardous situations at the bottom of the figure to more critically hazardous situations at the top. Between these two axes the suitability of these forms related to the degree of outsourcing is shown as darker shading for moderately outsourced IS and lighter shading for less moderately outsourced IS. This illustrates the suitability of networked and matrix organizational forms for certain outsourcing settings. 


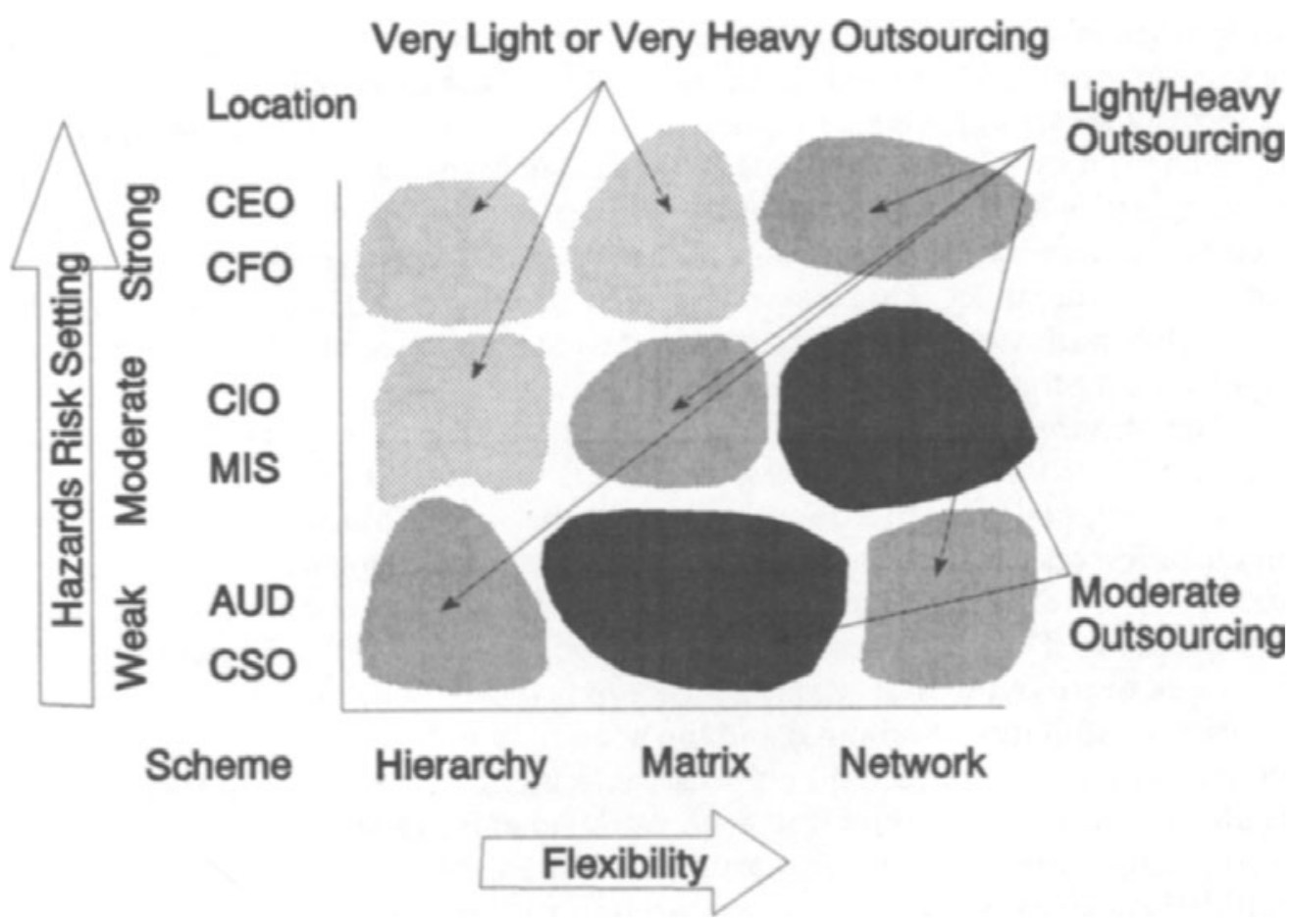

Figure 4. Dimensional analysis of hybrid IS security organizational forms.

There are two additional important notes about Figure 4. It would be unusual to plot an entire IS security organization as a single point intersecting the horizontal and vertical axes. It is more likely that the organization will be a hybrid of several different organizational forms. For example, the auditing functions might be characterized by the lower left-hand quadrant of Figure 4 while the program planning functions might be characterized by the upper right-hand quadrant. Our second note regards the shading. Figure 4 should not be read to imply that network and matrix forms are only suitable in outsourced settings. Rather, the shading only suggests that a best-practice characterization of an outsourced IS security function is motivated in part by the degree of outsourcing inherent in that function.

\section{CONCLUSION}

There are a large variety of ways in which an information systems security function might be structured. The most suitable solution is dependent on the organizational culture and its needs. But this does not mean that the various organizational forms are beyond analysis, and that there are no dimensions along which best practices might be positioned.

Examples of several important dimensions have been analyzed here: the functional inventory, organizational location, organizational scheme, and degree of 
outsourcing. Generalized advantages and disadvantages are distinguishable along each dimension. In addition, the importance of recognizing the hybrid nature of IS security organizational forms has been raised. While the discussion has been somewhat idealized, one major implication is that an IS security program cannot be fully developed, nor its full inventory of functions properly coordinated, until the nature of the IS organizational form is understood and planned. Without this understanding and planning, security functions cannot be properly managed and organizational information security will remain weakened. To this degree, proper IS security organizational planning and management is an important and highpriority IS security safeguard.

\section{REFERENCES}

Applegate, L. M. (1994) "Managing in an information age: Transforming the organization for the 1990s", in Baskerville et al. (eds) Transforming Organizations with Information Technology. Amsterdam: North-Holland, pp. 15-94.

Baskerville, R. (1993) "Information Systems Security: Adapting To Survive," Information Systems Security 2 (1) (Spring), pp. 40-47.

Forcht, K.A. (1994) Computer Security Management, Danvers, Massachusetts: Boyd \& Fraser

Hammer, M. and Champy, J. (1993) Reengineering the Corporation: A Manifesto for Business Revolution. New York: Harper-Collins.

Smithson, S.; Baskerville, R. and Ngwenyama, O. (1994) "Perspectives on Information Technology and New Emergent Forms of Organizations," in R. Baskerville, S. Smithson, O. Ngwenyama and J. DeGross (Eds.) Transforming Organizations with Information Technology. Amsterdam: North-Holland, 3-13.

\section{BIOGRAPHY}

Richard Baskerville is an associate professor in the School of Management at Binghamton University. His research focusses on security and methods in information systems, their interaction with organizations and research methods. He is an associate editor of MIS Quarterly and The Information Systems Journal. Baskerville's practical and consulting experience includes advanced information system designs for the U.S. Defense and Energy Departments. He is chair of the IFIP Working Group 8.2, a Chartered Engineer under the British Engineering Council, and holds MSc and PhD degrees from the London School of Economics. 